The Egyptian Journal of Hospital Medicine (July 2021) Vol. 84, Page 2308-2312

\title{
Evaluating the Role of Calprotectin in Pathogenesis of Acne Vulgaris in Zagazig University Hospital
}

Mohamed M. Nasr'1, Sharar Al Mokadem¹, Samar A Ahmed ${ }^{1}$, Heba E AbdEIrahman²

1-Dermatology, Venereology and Andrology Department, 2-Department of Clinical Pathology, Faculty of Medicine, Zagazig University, Egypt.

\section{ABSTRACT}

Background: Acne vulgaris is a chronic inflammatory disease of the pilosebaceous unit. The cardinal pathogenic factors include increased sebum secretion, follicular hyperkeratinization and microbial colonization by Cutibacterium acnes. Calprotectin is released from activated neutrophils, and can be detected in serum or other body fluids.

Aim: The aim of the present study was to clarify if calprotectin plays a role in acne vulgaris pathogenesis.

Subject and Methods: This study is a case control study included 42 patients complaining of acne vulgaris compared with42 apparent healthy individuals matching in age and sex with control group. Human calprotectin level in the serum was measured using enzyme linked immunosorbent assay (ELISA).

Results: The results showed that serum calprotectin level was higher in patients than controls but the difference was statistically insignificant $(\mathrm{P}=0.12)$. However, there was statistically significant correlation between serum calprotectin level in severe acne subtype and mild to moderate subtypes $(\mathrm{P}=0.026)$. The patients with acute onset had a higher serum calprotectin level than the patients with gradual onset $(\mathrm{P}=0.42)$, the difference was statistically insignificant. The male acne patients had higher statistically significant serum calprotectin level than female patients $(\mathrm{P}=0.03)$

Conclusion: Serum calprotectin level was higher in patients with acne vulgaris than controls, and is positively correlated with disease severity, therefore, might help in the evaluation of acne vulgaris severity and can help to evaluate the response to treatment.

Keywords: Acne vulgaris, Calprotectin

\section{INTRODUCTION}

Acne vulgaris is a cutaneous chronic inflammatory disease of the pilosebaceous unit affecting adolescents and is among the most common dermatological conditions worldwide. Acne is considered a chronic disease owing to its prolonged course, pattern of recurrence, relapse, and manifestations such as acute outbreaks or slow onset ${ }^{(\mathbf{1})}$.

Acne vulgaris has a complex pathogenesis. There were four factors that play a vital roles in acne pathophysiology, such as: hyperseborrhea and dysseborrhea, altered keratinization of the pilosebaceous duct, Cutibacterium acnes (C. acnes) and inflammation. The main hormones responsible for the development of acne vulgaris include androgens, insulin and insulin-like growth factor- $1^{(2)}$. The sequence in which these events occur is still not certain; inflammation has been suggested as the initial factor. The mechanisms behind the beginning and maintenance of the inflammatory response are not fully known, but Propionibacterium (Cutibacterium) acnes plays an important role in these mechanisms ${ }^{(\mathbf{3})}$. Cutibacterium acnes activates the innate immunity via the expression of protease activated receptors (PARs), tumor necrosis factor (TNF)- $\alpha$ and toll-like receptors (TLRs), which leads to production of interferon (INF)- $\gamma$, interleukins (IL8, IL12, IL-1), TNF, and matrix metalloproteinases (MMPs) by keratinocytes, resulting in the hyperkeratinization of the pilosebaceous unit ${ }^{(4,5)}$.

Calprotectin is the heterodimer form of S100A8 and S100A9, which are members of the S100 protein family. It plays a role in various inflammatory processes. Previously, calprotectin was also called calgranulin (due to its calcium binding property and it is mostly found in granulocytes), leukocyte-derived protein, cystic fibrosis antigen, and migration inhibitory factor-related proteins 8 and $14^{(6)}$ Calprotectin can be detected in many body fluids, including serum, saliva, synovial fluid and feces. Serum calprotectin levels increase in various cancers, inflammatory and autoimmune diseases ${ }^{(7)}$. It plays a role in infections, leukocyte migration, and cytoskeleton regulation. In addition, calprotectin binds to $\mathrm{Zn}^{2+}$ and $\mathrm{Mn}^{2+}$, which are metal nutrients for bacteria, and this binding produces a direct antimicrobial effect ${ }^{(\mathbf{8})}$.

Calprotectin has been found to be more accurate than other inflammatory markers, such as C-reactive protein (CRP) and erythrocyte sedimentation rate (ESR). Since calprotectin can be detected with minimal inflammation and it normalizes prior to CRP and ESR, it can be used to follow up of disease activity through the evaluation of treatment response or disease relapse, especially with autoimmune disorders ${ }^{(\mathbf{7 , 9})}$.

The aim of this study was to determine the calprotectin serum level in acne vulgaris patients and detect the correlation of its level with acne severity.

\section{SUBJECTS AND METHODS Subjects}

Forty-two acne vulgaris patients were included in this comparative case control study. All patients were collected from Dermatology, Andrology and 
Venereology Outpatient Clinic, Faculty of Medicine, Zagazig University Hospitals Human calprotectin level in the serum was measured in Clinical Pathology Department, Research Unit, Faculty of Medicine, Zagazig University Hospitals in the period from March2019 to August 2019. Forty-two apparently healthy subjects were selected of matching age and sex as possible and were taken as controls.

\section{Ethical approval:}

This case control study was conducted after approval of the Ethical Committee Board, Zagazig University and informed written consent from each patient was obtained. This work has been carried out in accordance with The Code of Ethics of the World Medical Association (Declaration of Helsinki) for studies involving humans.

The participants were classified into two main groups: Patient group: which was subdivided into three subgroups according to the global acne grading system(GAGS) ${ }^{(\mathbf{1 0})}$.Subgroup I: fourteen patients with mild acne vulgaris, Subgroup II: fourteen patients with moderate acne vulgaris and Subgroup III: fourteen patients with severe acne vulgaris. Control group: Forty two apparent healthy individuals; age and sex matched. Patients with a systemic disease, e.g. diabetes mellitus, heart failure, cirrhosis, infection, renal failure, or malignancy or with endocrinopathies (such as Cushing's syndrome or hyper- and hypothyroidism) and patients who had been using non-steroid anti-inflammatory drugs or hormonal medicines (such as steroids) in the last 3 months were excluded from the study.

Personal history including name, age, sex, marital status, residence, educational grade/occupation as well as special habits were taken from patients and control. Detailed medical history of the disease such as history of present illness including the onset, course and duration. Past history of previous treatment for acne (topical or systemic). Family history for the presence of acne vulgaris. General examination of body systems were performed to discover associated medical conditions. Detailed dermatological examination of the patients: Complete skin examination: including type and sites of acne. Acne severity was assessed by global acne grading system ${ }^{(\mathbf{1 0})}$.

\section{Method:}

Human calprotectin level in the serum was measured using enzyme linked immunosorbent assay (ELISA). $2 \mathrm{ml}$ of venous blood were collected from all patients and control group in plain tubes, allowed to clot at room temperature and then centrifuged at $10.000 \mathrm{rpm}$ for 10 min. Serum was separated and stored immediately at $20^{\circ} \mathrm{C}$ until assay of human calprotectin. Serum calprotectin was measured by standard double antibody sandwich enzyme-linked immunosorbent assay (ELISA) technique (Human Calprotectin ELISA Kit, Sun Red, Shanghai, China).The human Calprotectin ELISA is a ready-to-use solid-phase enzyme-linked immunosorbent assay. Patient, control samples and standards were added directly to wells of amicrotiter plate that is coated with antibody to calprotectin. After an incubation of a short period, the plate was washed and horseradish peroxidase (HRP) conjugated human calprotectin specific monoclonal antibody was added to each well. After incubation period for the second time, a "sandwich" of solid-phase antibody-human calprotectin-HRP conjugated monoclonal antibody" was formed. The unbound monoclonal antibodies and buffer matrix were removed in the subsequent washing step. The immunocomplex was detected by incubation with a substrate solution in a timed reaction and then measured in a spectrophotometric microplate reader. The enzymatic activity of the immunocomplex bound to the wall of each microtiter well in the microtiter plate is directly proportional to the amount of human calprotectin in the test sample. The enzyme reaction was stopped by the addition of oxalic acid (stop solution). The absorbance at $450 \mathrm{~nm}$ was measured by using spectrophotometer. A standard curve was obtained by plotting the absorbance (linear) versus the corresponding concentrations of the human calprotectin standards (log). The human calprotectin concentration of samples was determined from the standard curve.

\section{Statistical Analysis}

All data were collected and statistically analyzed using SPSS 20.0 for windows (SPSS Inc., Chicago, IL, USA 2011). Numerical data were expressed as a mean \pm SD and median, and range. Also, qualitative data were expressed as frequencies and percentage. Continuous data were checked for normality by using Shapiro Wilk test. Mann Whitney $U$ test was used for not-normally distributed quantitative data. The Kruskal-Wallis $\mathrm{H}$ test (sometimes also called the "one-way ANOVA on ranks") is a rank-based nonparametric test that can be used to determine if there are statistically significant differences between two or more groups of an independent variable . Post hoc Tukey test was used to detect significance of pairs of groups. P-value $<0.05$ was considered statistically significant.

\section{RESULTS}

The study included 42 patients and 42 healthy subjects, the mean age of patients was $17.95 \pm 2.84$ years and of controls was $19.57 \pm 2.69(\mathrm{P}=0.65)$. The patients included $18(42.9 \%)$ males and $24(57.1 \%)$ females and controls included $10(23.8 \%)$ males and 32 (76.2\%) females $(\mathrm{P}=0.19)$. There was no significant difference between both groups. 
Table (1) shows the clinical criteria of acne vulgaris of the patients.

\begin{tabular}{|c|c|c|}
\hline Items & & \\
\hline \begin{tabular}{|c|} 
Disease duration / month \\
Mean \pm SD \\
Median(range) \\
\end{tabular} & \multicolumn{2}{|c|}{$\begin{array}{c}24 \pm 18 \\
24(1-72)\end{array}$} \\
\hline & No & $\%$ \\
\hline $\begin{array}{l}\text { Onset of acne lesion } \\
\text { Acute } \\
\text { Gradual }\end{array}$ & $\begin{array}{c}6 \\
36\end{array}$ & $\begin{array}{l}14.3 \\
85.7\end{array}$ \\
\hline \begin{tabular}{|c|} 
Course of acne lesion \\
Progressive \\
Stationary \\
\end{tabular} & $\begin{array}{c}38 \\
4 \\
\end{array}$ & $\begin{array}{c}90.5 \\
9.5 \\
\end{array}$ \\
\hline \begin{tabular}{|l} 
Previous treatment \\
No ttt \\
Systemic $(1 \mathrm{y})$ \\
Topical ttt \\
Topical and systemic $\mathrm{ttt}(1 \mathrm{y})$
\end{tabular} & $\begin{array}{c}22 \\
2 \\
16 \\
2\end{array}$ & $\begin{array}{c}52.4 \\
4.8 \\
38 \\
4.8\end{array}$ \\
\hline \begin{tabular}{|l} 
Severity of acne lesions \\
Mild \\
Moderate \\
Severe
\end{tabular} & $\begin{array}{l}14 \\
14 \\
14\end{array}$ & $\begin{array}{l}33.3 \\
33.3 \\
33.3\end{array}$ \\
\hline
\end{tabular}

Y=year $\quad \mathrm{SD}=$ standard deviation $\quad \mathrm{No}=$ number $\quad \mathrm{tt}=$ treatment

Male patients had significantly higher serum calprotectin level than female patients.The difference was insignificant in the control group between males and females. In addition male patients had statistically higher level than male healthy controls (Table2).

Table(2): Comparison between both groups regarding serum calprotectin level and relation between its level and sex of patients and control

\begin{tabular}{|l|c|c|c|c|}
\hline \multicolumn{1}{|c|}{ Item } & $\begin{array}{c}\text { Patient group } \\
(\mathbf{n = 4 2})\end{array}$ & $\begin{array}{c}\text { Control group } \\
(\mathbf{n = 4 2})\end{array}$ & MW & P-Value \\
\hline $\begin{array}{l}\text { Calprotectin } \\
\text { serum level } \\
\text { (ng/ml) }\end{array}$ & & & & \\
Mean \pm SD & $2.31 \pm 0.04$ & $1.89 \pm 0.25$ & 1.58 & 0.12 \\
Median & 1.95 & 1.68 & \\
\hline \multicolumn{1}{|c|}{ Sex } & & & & \\
\hline Female & $1.9 \pm 0.31$ & $1.9 \pm 0.38$ & 0.023 & 0.98 \\
Mean \pm SD & 1.83 & 1.79 & & \\
Median & & & & \\
\hline Male & $2.86 \pm 0.26$ & $1.87 \pm 0.28$ & 2.14 & \\
Mean \pm SD & 2.49 & 1.46 & & \\
Median & 2.1 & 0.98 & & \\
\hline MW & $\mathbf{0 . 0 3 3}$ & 0.33 & & \\
P-value & SD & & & \\
\hline
\end{tabular}

MW =Mann WhitneyU test, $\mathrm{SD}=$ standard deviation, $\mathrm{n}=$ number.

The highest level of calprotectin in serum was recorded in subgroup of severe acne. The difference between the subgroups was statistically significant (Table 3).

Post hoc test shows statistically significant difference between severe acne type and mild, moderate subtypes, $\mathrm{P}=0.027, \mathrm{P}=0.013$ respectively and there was statistically insignificant difference between mild and moderate subtypes, $\mathrm{P}>0.05$. 
Table (3): Relation of mean of calprotectin serum level with the subgroups of patients

\begin{tabular}{|c|c|c|c|c|c|}
\hline \multirow{2}{*}{ Item } & \multicolumn{3}{|c|}{ Subgroups } & \multirow{2}{*}{$\begin{array}{c}\text { Kruskal-Wallis } \\
\text { Test }\end{array}$} & \multirow{2}{*}{ P-value } \\
\hline & $\begin{array}{l}\text { Mild } \\
n=14\end{array}$ & $\begin{array}{c}\text { Moderate } \\
\mathrm{n}=14\end{array}$ & $\begin{array}{c}\text { Severe } \\
n=14\end{array}$ & & \\
\hline $\begin{array}{l}\text { Calprotectin } \\
\text { serum level }(\mathrm{ng} / \mathrm{ml}) \\
\text { Mean } \pm \text { SD } \\
\text { Median }\end{array}$ & $\begin{array}{c}1.99 \pm 0.26 \\
1.88\end{array}$ & $\begin{array}{c}1.82 \pm 0.26 \\
1.78\end{array}$ & $\begin{array}{c}3.14 \pm 0.29 \\
2.92\end{array}$ & 6.51 & 0.026 \\
\hline
\end{tabular}

$\mathrm{SD}=$ standard deviation, $\mathrm{n}=$ number

In patients with disease duration $>6$ months, acute onset and progressive course of the disease, serum calprotectin level was higher than in patients with disease duration $\leq 6$ months, gradual onset or Stationary course of the disease ,but with no significant statistically difference (table 4).

Table (4): Relation between serum calprotectin level of patients with onset, course and duration of disease

\begin{tabular}{|c|c|c|c|c|c|c|}
\hline \multirow{2}{*}{ Items } & \multicolumn{4}{|c|}{ Serum calprotectin level } & \multirow{2}{*}{ MW } & \multirow[b]{2}{*}{ P-value } \\
\hline & No & $\%$ & Mean \pm SD & Median (range) & & \\
\hline $\begin{array}{l}\text { Disease duration } \\
\leq 6 \text { months } \\
>6 \text { months }\end{array}$ & $\begin{array}{c}8 \\
34\end{array}$ & $\begin{array}{l}18.1 \\
80.9\end{array}$ & $\begin{array}{l}1.78 \pm 0.66 \\
2.43 \pm 1.1\end{array}$ & $\begin{array}{l}1.69(1.09-2.64) \\
1.97(1.21-5.61)\end{array}$ & 1.16 & 0.24 \\
\hline $\begin{array}{l}\text { Onset } \\
\text { Acute } \\
\text { Gradual }\end{array}$ & $\begin{array}{c}6 \\
36 \\
\end{array}$ & $\begin{array}{l}14.3 \\
85.7\end{array}$ & $\begin{array}{l}3.27 \pm 2.18 \\
2.15 \pm 0.72 \\
\end{array}$ & $\begin{array}{l}2.92(1.29-5.61) \\
1.94(1.09-3.74) \\
\end{array}$ & 0.80 & 0.42 \\
\hline $\begin{array}{l}\text { Course } \\
\text { Progressive } \\
\text { Stationary }\end{array}$ & $\begin{array}{c}38 \\
4\end{array}$ & $\begin{array}{c}90.5 \\
9.5\end{array}$ & $\begin{array}{l}2.4 \pm 1.48 \\
1.49 \pm 0.55\end{array}$ & $\begin{array}{c}1.98(1.21-5.61) \\
1.49(1.09-1.88)\end{array}$ & 1.55 & 0.12 \\
\hline
\end{tabular}

MW=Mann-Whitney U test, no=number, $\mathrm{SD}=$ standard deviation 
https://ejhm.journals.ekb.eg/

\section{DISCUSSION}

The current study was conducted to evaluate the relationship between acne vulgaris and serum level of calprotectin and compare its level in both patients and apparent healthy individuals.

Our results showed that the calprotectin serum level was higher in patients than controls but the difference was statistically insignificant. This came in contrast with the results shown by Korkmaz and Fiçıcıoğlu ${ }^{(11)}$ and Fouda et $\boldsymbol{a l l}^{(\mathbf{1 2})}$, who have found a significant higher serum calprotectin level of patients than healthy controls.

In this study, there was statistically significant difference in serum level between severe acne subtype and mild to moderate subtypes while there was statistically insignificant difference between mild and moderate subtypes. In previous studies carried out by Korkmaz and Fiçıcıoğlu ${ }^{(11)}$ and Fouda et $\boldsymbol{a l}^{(\mathbf{1 2})}$,the serum calprotectin level of the moderate and severe groups were significantly higher than that of the mild group. In addition, the serum calprotectin level of the mild acne vulgaris group was significantly higher than that of the healthy control group.

The serum calprotectin level was higher in cases with disease duration $>6$ months than patients with disease duration $\leq 6$ months, however, this difference was statistically insignificant. In patients with progressive disease course, the level was higher than patients with stationary disease course, but the difference was statistically insignificant.The patients with acute onset hada higher level of serumcalprotectin than the patients with gradual onset, but it was statistically insignificant. However the study carried out by Fouda et al. ${ }^{(\mathbf{1 2})}$ showed that, there was a significant positive correlation between serum levels of calprotectin and duration of the disease.

The current study demonstrated that male acne patients had a significant higher serum calprotectin level than female patients, whereas there was insignificant difference between males and females of control group. In addition, male patients compared to male healthy controls have statistically significant higher level of serum calprotectin.

To our knowledge very limited number of studies discussed the relationship between calprotectin and acne vulgaris (Korkmaz and Fıçıcıoğlu ${ }^{(11)}$ and Fouda et $\left.\boldsymbol{a l}{ }^{(\mathbf{1 2})}\right)$. The difference between the current study and previous studies may be explained by different sample size and/or different ethnic group.

The results of studies carried out by Korkmaz and Fiçıcıoğlu ${ }^{(11)}$ and Fouda et $\boldsymbol{~ a l . ~}{ }^{(\mathbf{1 2})}$ revealed that calprotectin serum level was higher in patients than controls, in severe cases and with longer duration. Our results were to some extent similar to these findings but with no significant relations. This could be explained by the wide variation between patients and the complexity of acne pathogenesis.
From previous results we can conclude that calprotectin is involved in some aspects to inflammatory processes of acne vulgaris. The higher level in serum of patients than controls, the higher level in severe subtype than mild to moderate subtype, the higher level in male patients than female patients, the higher level in male patients than male healthy controls, the higher level with prolonged duration and the higher level with progressive disease course than stationary course indicate that calprotectin is one factor among many other interacting factors mediating the pathogenesis and severity of acne vulgaris. Further larger studies are recommended to emphasize the role of calprotectin as a biomarker in the evaluation of disease severity, response to treatment and its role in pathogenesis of acne vulgaris.

\section{REFERENCES}

1- MoradiTuchayi S, Makrantonaki E, Ganceviciene $\mathbf{R}$ et al.(2015):Acne vulgaris. Nature reviews. Disease primers, 1:15029.

2- Suh H, Kwon H (2015): What's new in the physiopathology of acne? The British journal of dermatology, 172 (1):13-19.

3- Bhat J, Latief I, Hassan I (2017):Update on etiopathogenesis and treatment of Acne. Indian journal of dermatology, venereology and leprology, 83(3):298-306.

4- Gollnick P (2015): From new findings in acne pathogenesis to new approaches in treatment. Journal of the European Academy of Dermatology and Venereology, 29 Suppl, 5: 17.

5- Su Q, Grabowski M, Weindl G (2017):Recognition of Propionibacterium acnes by human TLR2 heterodimers. International journal of medical microbiology, 307(2): 108-112.

6- Bojko J (2017):Measurement of blood calprotectin (MRP8/MRP-14) levels in patients with juvenile idiopathic arthritis. Reumatologia, 55(1): 10-14.

7- De PontiA, Wiechert L, Stojanovic A et al.(2015): Chronic liver inflammation and hepatocellular carcinogenesis are independent of S100A9. Int. J. Cancer, 136: 2458-2463.

8- Nishikawa Y, Kajiura Y, Lew H et al.(2017): Calprotectin Induces IL-6 and MCP-1 Production via Toll-Like Receptor 4 Signaling in Human Gingival Fibroblasts. Journal of cellular physiology, 232(7):1862-1871.

9- Berg-Hansen P, Vandvik B, Fagerhol M et al.(2009): Calprotectin levels in the cerebrospinal fluid reflect disease activity in multiple sclerosis. Journal of neuroimmunology, 216(1-2): 98-102.

10- Doshi A, Zaheer A, Stiller J (1997):A comparison of current acne grading systems and proposal of a novel system. International journal of dermatology, 36(6): 416-418.

11- Korkmaz S, Fiçıcıoğlu S (2018):Calprotectin can play an inflammatory role in acne vulgaris .Postępy Dermatol Alergologl., 35: 397 - 399.

12- Fouda F, Obaid M, Hegazy F et al.(2021):Calprotectin in acne vulgaris: A possible contributory role. J CosmetDermatol., 20: 621-625. 